\title{
Optimization of Uplink CSI Training for Full-Duplex Multiuser MIMO Systems
}

\author{
Author 1, Author 2, Author 3 and Author 4
}

\begin{abstract}
In this letter, we investigate the optimization of uplink (UL) channel state information (CSI) training in the full-duplex (FD) based multiuser (MU) multiple-input multipleoutput (MIMO) systems. Here, an FD-assisted BS performs simultaneous UL CSI training and downlink data transmission that produces self interference (SI). Following the UL training stage, the BS uses the conventional half-duplex (HD) MU MIMO transmission with zero-forcing precoding. To find an optimal UL CSI training length that achieves a balance between the training overhead and CSI quality, we formulate an optimization problem that maximizes the sum spectral efficiency of the network. To ease the analysis, we derive a lower bound on the user rate in the FD phase, which is then used together with the HD user rate to obtain the sub-optimal solution. We also provide a closed-form expression to approximate the UL training length. Numerical results show that the performance of the proposed UL training outperforms the fixed length training and it closely matches the performance with an exhaustive search.
\end{abstract}

Index Terms-MIMO communication, precoding

\section{INTRODUCTION}

$\mathbf{R}$ ECENTLY, full duplex (FD) capabilities are explored to acquire channel state information (CSI) from users at a base station (BS) in a spectrally efficient manner [1]. Such systems [1], [2] have shown to provide a significant spectral efficiency gain in multiuser (MU) multiple-input multipleoutput (MIMO) systems. Two main factors limiting the performance of these integrated systems are self interference (SI) and CSI training overhead. Nevertheless, by employing SI cancellation techniques, the residual SI can be brought down to a noise level. SI cancellation techniques in MIMO systems are based on analog [3], digital [4] or combination of the both cancellation schemes [5]. More insightful discussion on SI cancellation is provided in [6].

Uplink (UL) CSI training in time division duplex (TDD) based MU MIMO systems is crucial for implementing MU precoding, but it consumes significant amount of channel uses within the coherence interval. Moreover, UL CSI training plays an important role in determining the spectral efficiency of the MU MIMO system. This factor determines two important performance aspects: i) the quality of CSI at the BS and ii) the amount of time slots for the actual data transmission. Hence, it is important to judiciously design the UL CSI training length such that a balance is achieved between the training overhead and the CSI quality.

Previous studies have investigated CSI training optimization for TDD based MIMO systems, assuming block fading channels, for both single user (SU) [7], [8] and MU [9], [10] MIMO systems. The UL training for SU MIMO was first investigated in [7], where the lower bounded capacity of the system was maximized to obtain an optimal UL training duration. According to [7], the optimum number of required training symbols becomes equal to the number of transmit antennas at the BS. For MU MIMO broadcast systems, the optimal UL CSI training with zero-forcing (ZF) precoding is thoroughly investigated in [9]. Here, the optimal UL CSI training is achieved by maximizing the lower bounded achievable rate of a user. It has been reported in [9] that the optimal training depends on the total number of transmit antennas and coherence interval.

The aforementioned seminal studies are based on conventional half duplex (HD) MIMO systems. In this work, we determine the optimum fraction of the transmission interval that should be allocated to the UL CSI training in the case of an FD assisted BS and in the presence of residual SI, which has not been studied before in the literature. In [1], authors investigate optimal training for FD MU MIMO systems assuming perfect CSI at users and no residual SI at the BS. Whereas, in this study, we rely on statistical knowledge of the effective channel for the coherent detection at the user. Moreover, we consider that residual SI is also present in the system. Here, similar to [2], simultaneous downlink (DL) data transmission and UL CSI reception at the BS is achieved in the same time-frequency resource block. We use the lower bounded achievable rates derived for the ZF precoding scheme in [2] for both FD and HD phases to obtain the CSI training length. This work is an extension of [2], where the optimization of UL CSI training was not investigated and only fixed length training was assumed.

In this paper, we provide the analysis for optimizing the UL CSI training such that the sum rate (in FD and HD phases) of the system is maximized. To simplify the derivation of the UL training length, we use the lower bounded FD rate and use it together with the HD rate as an objective function to obtain the sub-optimal solution for the UL CSI training length. Due to the concavity of the objective function, we rely on the (root finding) Brent's method to find the sub-optimal CSI training length from the complicated function obtained after taking the derivative of the objective function. Furthermore, we also provide a closed form expression to approximate the UL training length.

\section{SySTEM MOdEL}

We consider an FD-assisted BS equipped with two sets of antennas, i.e., $M_{t}$ transmitting antennas and $M_{r}$ receiving antennas. The BS serves $K$ single-antenna users simultaneously using ZF precoding. Similar to [2], [9], it is assumed that the UL training consumes downlink (DL) channel uses. We consider a block Rayleigh fading channel model, where the 


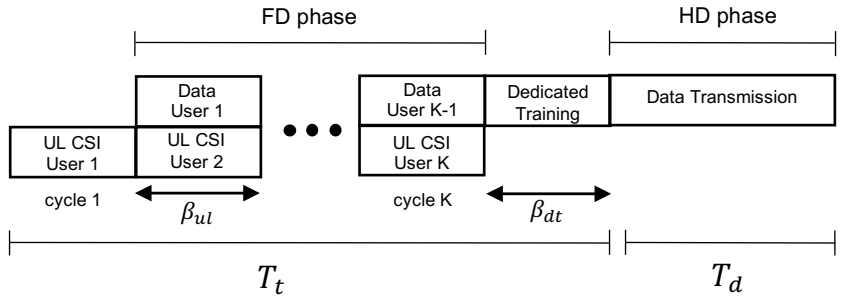

Fig. 1: An illustration of a coherence block of $T=T_{t}+T_{d}$ channel uses with FD and HD phases.

channel value remains constant over the coherence block of $T$ channel uses. Here, without loss of generality, we assume that $M_{t}=M_{r}=M$. There are two main transmission phases: i) FD phase and ii) HD phase. The FD phase consists of simultaneous UL pilot reception and DL data transmission to the specific subset of scheduled users, whereas, the HD phase includes the DL data transmission to all the $K$ users. The UL CSI training and DL data transmission within a block length $T$ is shown in Fig. 1, where $T$ is equal to the sum of channel uses for UL training, $T_{t}$, and DL data transmission, $T_{d}$. The length of the UL pilot sequence is denoted by $\beta_{\mathrm{ul}}$, whereas, the length of the dedicated training sequence (for the purpose of coherent detection) at the BS is represented by $\beta_{\mathrm{dt}}$. We assume that the SI is mitigated using a suitable SI cancellation technique, however, there is only a residual SI present in the system. Due to the space limitation, we refer the reader to [2] for a detailed and comprehensive description of the system model. Here, we briefly discuss the details of FD and HD phases.

FD phase: Each user transmits $\beta_{\mathrm{ul}}$ number of UL pilot symbols to the BS. There are total $K$ cycles and each cycle consumes $\beta_{\mathrm{ul}}$ channel uses. The received signal at the BS from the $k^{\text {th }}$ user during the UL training is given by ${ }^{1}$ $\mathbf{Y}_{k}=\sqrt{\beta_{\mathrm{ul}} p_{k}} \mathbf{h}_{k} \mathbf{s}^{T}+\mathbf{\Psi}+\mathbf{Z}_{k}$, where $p_{k}$ is the transmit power of the $k^{\text {th }}$ user, $\mathbf{s}$ denotes the UL pilot sequence, such that $\mathbf{s} \in \mathbb{C}^{\beta_{\text {ul }} \times 1}$. The Rayleigh fading channel between the $k^{\text {th }}$ user and the BS is given by $\mathbf{h}_{k}$, where $\mathbf{h}_{k} \in \mathcal{C N}(0, \mathbf{I})$ and $\mathbf{Z}_{k}$ is the noise matrix of size $M \times \beta_{\mathrm{ul}}$ whose entries follow $\mathcal{C N}\left(0, N_{0}\right)$ distribution. The residual SI matrix at the BS during UL training is given by $\boldsymbol{\Psi}=\left[\boldsymbol{\psi}_{1}, \cdots, \boldsymbol{\psi}_{\beta_{\mathrm{ul}}}\right]$ with $\boldsymbol{\psi}_{n}=\mathbf{H}_{\mathrm{SI}} \mathbf{V}_{c} \mathbf{u}_{n}$, where the residual SI channel of size $M \times M$ is denoted by $\mathbf{H}_{\mathrm{SI}}$ whose entries follow the $\mathcal{C N}\left(0, \nu^{2}\right)$ distribution. The variance of the residual SI channel is given by $\sigma_{\mathrm{SI}}^{2}=\nu^{2}$. The precoding matrix in the $c^{\text {th }}$ cycle is given by $\mathbf{V}_{c} \in \mathbb{C}^{M \times j}$, where $j$ denotes the number of users being served by the BS in the $c^{\text {th }}$ cycle, such that $j=c-1$. The vector $\mathbf{u}_{n} \in \mathbb{C}^{j}$ comprises of the independent data symbols and it follows the $\mathcal{C N}(0, P \mathbf{I})$ distribution. After de-spreading $\mathbf{Y}_{k}$ at the BS, we get $\mathbf{r}_{k}=\mathbf{Y}_{k} \mathbf{s}^{*}$, which can be expressed as

$$
\mathbf{r}_{k}=\sqrt{\beta_{\mathrm{ul}} p_{k}} \mathbf{h}_{k}+\tilde{\boldsymbol{\psi}}+\tilde{\mathbf{z}}_{k},
$$

where $\tilde{\mathbf{z}}_{k}=\mathbf{Z}_{k} \mathbf{s}^{*} \in \mathcal{C N}\left(0, N_{0} \mathbf{I}\right)$ and $\tilde{\boldsymbol{\psi}}=\mathbf{\Psi} \mathbf{s}^{*}$. For the first scheduled user, we have $\tilde{\psi}=0$, i.e., no residual SI. The

\footnotetext{
${ }^{1}$ We use $(\cdot)^{H},(\cdot)^{*},(\cdot)^{T},(\cdot)^{-1}$ and $(\cdot)^{\perp}$ to denote the conjugate transpose, the conjugate, the transpose, the inverse and the pseudoinverse operations, respectively. $\mathbb{E}[\cdot]$ denotes expectation. The complex normal distribution with mean $\mu$ and variance $\sigma^{2}$ is denoted by $\mathcal{C N}\left(\mu, \sigma^{2}\right)$.
}

BS estimates $\mathbf{h}_{k}$ by applying the minimum mean square error (MMSE) criterion to the observation $\mathbf{r}_{k}$, resulting in [2]

$$
\begin{aligned}
\hat{\mathbf{h}}_{k} & =\mathbb{E}\left[\mathbf{h}_{k} \mathbf{r}_{k}^{H}\right] \mathbb{E}\left[\mathbf{r}_{k} \mathbf{r}_{k}^{H}\right]^{-1} \mathbf{r}_{k} \\
& =\frac{\sqrt{\beta_{\mathrm{ul}} p_{k}}}{\beta_{\mathrm{ul}} p_{k}+N_{0}+P \nu^{2}} \mathbf{r}_{k} .
\end{aligned}
$$

The channel estimation error vector, $\mathbf{e}_{k}=\mathbf{h}_{k}-\hat{\mathbf{h}}_{k}$ has a covariance of $\mathbb{E}\left[\mathbf{e}_{k} \mathbf{e}_{k}^{H}\right]=\sigma_{\mathbf{e}_{k}}^{2} \mathbf{I}$, where

$$
\sigma_{\mathbf{e}_{k}}^{2}=1-\frac{\beta_{\mathrm{ul}} p_{k}}{\left(\beta_{\mathrm{ul}} p_{k}+N_{0}+P \nu^{2}\right)} .
$$

The variance of the estimated channel for the $k^{\text {th }}$ user denoted by $\sigma_{\hat{\mathbf{h}}_{k}}^{2}$ is $\sigma_{\hat{\mathbf{h}}_{k}}^{2}=1-\sigma_{\mathbf{e}_{k}}^{2}$. For $c>1$, the BS in each cycle serves $c-1$ users in the DL using the ZF precoding scheme, such that the received signal at the $k^{\text {th }}$ user can be written as

$$
y_{k}^{\mathrm{FD}}=\mathbf{h}_{k}^{H} \mathbf{x}_{c}+h_{q, k}^{\mathrm{ul}} s_{q}+n_{k},
$$

where $n_{k} \sim \mathcal{C N}\left(0, N_{0}\right)$ denotes the noise at the $k^{\text {th }}$ user. The transmitted signal from the BS is given by $\mathbf{x}_{c}=\mathbf{V}_{c} \mathbf{u}_{c}$, which is subject to the average power constraint of $\mathbb{E}\left[\left|\mathbf{x}_{c}\right|^{2}\right] \leq P$. A user $q$ (i.e., the next scheduled user) transmits its UL pilot symbols to the BS, thus, creating inter-node interference at the $k^{\text {th }}$ user. The interfering channel between the $q^{\text {th }}$ user and the $k^{\text {th }}$ user is denoted by $h_{q, k}^{\mathrm{ul}}$, where $h_{q, k}^{\mathrm{ul}} \sim \mathcal{C} \mathcal{N}(0,1)$ and $s_{q}$ is a pilot symbol of the $q^{\text {th }}$ user, where $\left|s_{q}\right|^{2}=1 / \beta_{\mathrm{ul}}$. The transmitted power of the $q^{\text {th }}$ user is denoted by $p_{q}$. The imperfect CSI at the BS in the cycle $c$ is given by $\hat{\mathbf{H}}_{c}=\left[\hat{\mathbf{h}}_{1}, \ldots, \hat{\mathbf{h}}_{j}\right]^{T}$. The ZF precoding vector of the $k^{\text {th }}$ user is denoted by $\mathbf{v}_{k}$, which is the $k^{\text {th }}$ normalized column of the matrix $\mathbf{V}_{c}$, where $\mathbf{V}_{c}=\hat{\mathbf{H}}_{c}^{H}\left(\hat{\mathbf{H}}_{c} \hat{\mathbf{H}}_{c}^{H}\right)^{-1}$.

HD phase: To equip the users with effective channel gain for coherent detection [11], dedicated training is performed, where the BS transmits $\beta_{\mathrm{dt}}$ pilots using the ZF precoding scheme. The $k^{\text {th }}$ user applies an orthogonal complement of the pilot matrix to the received pilot matrix to obtain the received pilot vector, given by $\overline{\mathbf{y}}_{k}=\sqrt{\beta_{\mathrm{dt}} P} \mathbf{a}_{k}+\mathbf{z}_{k}$, where $\mathbf{a}_{k}=\left[\begin{array}{llll}a_{k, 1} & a_{k, 2} & \cdots & a_{k, K}\end{array}\right]$ with $a_{k, i}=\mathbf{h}_{k}^{H} \mathbf{v}_{i}, i=1, \cdots, K$ and $\mathbf{z}_{k}$ has independent entries with the $\mathcal{C N}\left(0, N_{0}\right)$ distribution. The user $k$ estimates its useful channel coefficient using the MMSE estimate on the $k^{\text {th }}$ entry of $\overline{\mathbf{y}}_{k}$, yielding $\hat{a}_{k, k}=\sqrt{\beta_{\mathrm{dt}} P}\left(\beta_{\mathrm{dt}} P+N_{0}\right)^{-1} \bar{y}_{k, k}$ [11]. The variance of the error between the actual $a_{k, k}$ and estimated $\hat{a}_{k, k}$ is given by $\sigma_{\lambda_{k}}^{2}=N_{0} /\left(N_{0}+\beta_{\mathrm{dt}} P\right)$ and the variance of $\hat{a}_{k, k}$ is $1-\sigma_{\lambda_{k}}^{2}$. After dedicated training, the BS transmits the data symbols $\mathbf{u}=\left[u_{1}, \ldots, u_{K}\right]$ to all the $K$ users using the ZF precoding scheme till the end of the frame duration, $T$. The received signal at this stage is similar to (5) without inter-node interference with $\mathbf{x}=\mathbf{V u}$ and $\mathbf{V} \in \mathbb{C}^{M \times K}$.

Achievable rate: The achievable rate of the user in the network is the sum of the rates in FD and HD phases. From [1], [2], the rate of the $k^{\text {th }}$ user can be written as

$$
R=\underbrace{\frac{\beta_{\mathrm{ul}}}{K T} \sum_{c=2}^{K} \sum_{k=1}^{c-1} R_{k, c}^{\mathrm{FD}}}_{R^{\mathrm{FD}}}+\underbrace{\left(1-\frac{T_{t}}{T}\right) R_{k}^{\mathrm{HD}}}_{R^{\mathrm{HD}}},
$$

where $R_{k, c}^{\mathrm{FD}}$ is the FD rate of the $k^{\text {th }}$ user in the $c^{\text {th }}$ cycle and 
$R_{k}^{\mathrm{HD}}$ is the rate of the $k^{\text {th }}$ user in the HD phase. Using the results derived in [2], we can write the lower bounded rates $R^{\mathrm{FD}}$ and $R^{\mathrm{HD}}$ as a function of $\beta_{\mathrm{ul}}$, denoted by $\tilde{R}^{\mathrm{FD}}$ and $\tilde{R}^{\mathrm{HD}}$, respectively, given by ${ }^{2}$

$$
\begin{aligned}
& \tilde{R}^{\mathrm{FD}}\left(\beta_{\mathrm{ul}}\right) \triangleq \frac{\beta_{\mathrm{ul}}}{K T} \sum_{c=2}^{K}(c-1) \\
& \times \log _{2}\left(1+\frac{\frac{P}{N_{0}} \sigma_{\hat{\mathbf{h}}_{k}}^{2}(M-c+2)}{(c-1)\left(1+\frac{P}{N_{0}} \sigma_{\mathbf{e}_{k}}^{2}+\frac{p_{q}}{\beta_{\mathrm{ul}} N_{0}}\right)+\frac{P}{N_{0}}\left(\frac{\sigma_{\hat{\mathbf{h}}_{k}}^{2}}{4}-\sigma_{\mathbf{e}_{k}}^{2}\right)}\right) .
\end{aligned}
$$

and

$$
\begin{aligned}
\tilde{R}^{\mathrm{HD}}\left(\beta_{\mathrm{ul}}\right) & \triangleq\left(1-\frac{\left(K \beta_{\mathrm{ul}}+\beta_{\mathrm{dt}}\right)}{T}\right) \\
& \times \log _{2}\left(1+\frac{\frac{P}{K N_{0}} \frac{\beta_{\mathrm{dt}} P\left(\beta_{\mathrm{dt}} P(M-K+1)+N_{0}\right)}{\left(N_{0}+\beta_{\mathrm{dt}} P\right)^{2}}}{1+\frac{P}{K N_{0}} \sigma_{\lambda_{k}}^{2}+\frac{P}{K N_{0}}(K-1) \sigma_{\mathbf{e}_{k}}^{2}}\right) .
\end{aligned}
$$

It is important to choose the UL pilot sequence length optimally to achieve a balance between the CSI quality and training overhead. This balance is vital to achieve higher sum rates in the network. For instance, when $\beta_{\mathrm{ul}}$ is large, the FD rate increases, whereas, the HD rate decreases, however, the quality of CSI at the BS improves, and vice versa.

\section{UL CSI TRAINING OPTIMIZATION}

The sophisticated relationship between the UL CSI training length and achievable rate motivates us to investigate the optimal UL CSI training length that maximizes the sum rate of the system. Similar to [9], we do not consider the optimization of the DL training (dedicated training). The dedicated training is a one-off training which unlike UL CSI training does not scale with the number of users in the network, hence, not significantly effecting the performance of the system. As $K-1$ users are served in the FD phase and all the $K$ users are served in the HD phase, we can formulate the optimization problem to maximize the DL sum rate as

$$
\begin{array}{ll}
\max _{\beta_{\mathrm{ul}}} & (K-1) \tilde{R}^{\mathrm{FD}}\left(\beta_{\mathrm{ul}}\right)+K \tilde{R}^{\mathrm{HD}}\left(\beta_{\mathrm{ul}}\right) \\
& \text { s.t. } \quad 0 \leq \beta_{\mathrm{ul}} \leq T .
\end{array}
$$

To ease the analysis, we derive the lower bound for the FD sum rate, $\tilde{R}^{\mathrm{FD}}$, that is independent of the cycle number, $c$. It is evident from (7) that $\tilde{R}^{\mathrm{FD}}\left(\beta_{\mathrm{ul}}\right)$ is a monotonically increasing non-negative function of $c$. Therefore, we can lower bound the FD rate (7) by the rate in the first downlink cycle, which corresponds to the cycle number 2, i.e., $c=2$, with a single user whose CSI at the BS is assumed to be affected by the residual SI. The lower bounded FD sum rate is given by

$$
\begin{aligned}
(K-1) \tilde{R}^{\mathrm{FD}}\left(\beta_{\mathrm{ul}}\right) & \geq \hat{R}^{\mathrm{FD}}\left(\beta_{\mathrm{ul}}\right) \\
& =a \beta_{\mathrm{ul}} \log \left(1+\frac{\beta_{\mathrm{ul}} p_{k} \Delta}{\beta_{\mathrm{ul}} \eta+\delta+\frac{\epsilon}{\beta_{\mathrm{ul}}}}\right),
\end{aligned}
$$

\footnotetext{
${ }^{2}$ The lower bounded FD rate, $\tilde{R}^{\mathrm{FD}}$ in (7) assumes that the channel estimate of the user 1 is also affected by the residual SI.
}

where, $a=(K-1) /(K T \log (2)), \Delta=P M / N_{0}, \eta=p_{k}+$ $P p_{k} /\left(4 N_{0}\right), \gamma=N_{0}+P \nu^{2}, \epsilon=\gamma p_{q} / N_{0}$ and $\delta=\gamma+$ $\left(p_{q} p_{k}\right) / N_{0}$. The FD rate in (7) approaches the lower bounded rate (10) as $K \rightarrow 1$. For clarity, we can write the HD sum rate in (9) as

$$
\hat{R}^{\mathrm{HD}}\left(\beta_{\mathrm{ul}}\right) \triangleq \frac{\Lambda-K \beta_{\mathrm{ul}}}{T \log (2)} K \log \left(1+\frac{\Gamma}{\lambda-\frac{\gamma(2-K)}{\beta_{\mathrm{ul}} p_{k}+\gamma}}\right),
$$

where $\Lambda=T-\beta_{\mathrm{dt}}, \lambda=\sigma_{\lambda_{k}}^{2}+\left(K N_{0}\right) / P$ and $\Gamma=$ $\beta_{\mathrm{dt}} P\left(\beta_{\mathrm{dt}} P(M-K+1)+N_{0}\right)\left(\beta_{\mathrm{dt}} P+N_{0}\right)^{-2}$. Following the lower bounded rate $(10)$, we can rewrite the modified optimization problem, with $x$ denoting the sub-optimal UL training, as

$$
\begin{array}{cc}
\max _{x} & \hat{R}^{\mathrm{FD}}(x)+\hat{R}^{\mathrm{HD}}(x) \\
\text { s.t. } & 0 \leq x \leq T .
\end{array}
$$

Denoting the objective function in (12) by $\hat{R}(x)$, we note that the first derivative of $\hat{R}(x)$, given by $\hat{R}^{\prime}(x)$, is a monotonically decreasing function with respect to $x$, where $x \in\left[0, x_{f}\right]$ and $x_{f}$ is a real non-negative integer. This means that the objective function, $\hat{R}(x)$, is concave in $x$, and therefore, the UL pilot sequence length $x^{\star}$ can be obtained by solving

$$
\hat{R}^{\prime}(x)=\frac{\partial}{\partial x}\left[\hat{R}^{\mathrm{FD}}(x)+\hat{R}^{\mathrm{HD}}(x)\right]=0 .
$$

We begin by taking the derivative of the FD rate (10), yielding

$$
\frac{\partial \hat{R}^{\mathrm{FD}}(x)}{\partial x}=a \log \left(1+\frac{p_{k} \Delta x^{2}}{g(x)}\right)-\frac{a \eta x^{2}-a \epsilon}{g(x)}+a,
$$

where $g(x)=\eta x^{2}+\delta x+\epsilon$. Similarly, we take the derivative of the HD rate (11) with respect to $x$, giving

$$
\frac{\partial \hat{R}^{\mathrm{HD}}(x)}{\partial x}=-b \log \left(1+\frac{\Gamma v(x)}{h(x)}\right)+\left(\Omega-\frac{K x}{T}\right) \frac{p_{k} N K h(x)^{-1}}{v(x) \log (2)},
$$

where $b=K^{2} /(T \log (2)), \quad N=\gamma(K-1), v(x)=$ $\left(x p_{k}+\gamma\right), \Omega=1-\left(\beta_{\mathrm{dt}} / T\right)$ and $h(x)=\lambda\left(x p_{k}+\gamma\right)+N$. Now substituting (14) and (15) in (13), we get

$$
\begin{aligned}
& a \log \left(1+\frac{p_{k} \Delta x^{2}}{g(x)}\right)-\frac{a \eta x^{2}-a \epsilon}{g(x)}+a-b \log \left(1+\frac{\Gamma v(x)}{h(x)}\right) \\
& +\left(\Omega-\frac{K x}{T}\right) \frac{p_{k} N K h(x)^{-1}}{v(x) \log (2)}=0 .
\end{aligned}
$$

Solving (16) for $x$ yields the sub-optimal solution for the CSI training length $\beta_{\mathrm{ul}}^{\star}$ in (9). Note that $x$ provides the sub-optimal solution of the original problem (9) as we have used the lower bounded FD rate (10) in our analysis. It is cumbersome to explicitly express $x$ in (16) due to the complexity of the function. Therefore, in order to obtain $\beta_{\mathrm{ul}}^{\star}$, we rely on a root finding algorithm known as Brent's method. The Brent's method seeks a zero of a function within a given interval using the combination of three methods: the bisection method, the secant method and the inverse quadratic interpolation. It is reliable and fast converging algorithm. In this work, the solution of (16) lies in an interval $\left(x_{i}, x_{f}\right)$, where both points are real non-negative integers. We can also approximate (16) to obtain the closed-form solution for the CSI training length, 
as

$$
\tilde{\beta}_{\mathrm{ul}} \triangleq \frac{1}{2 \lambda p_{k}^{2}}\left[-\tau+\sqrt{\tau^{2}-4 \lambda p_{k}^{2} \varphi}\right]
$$

where

$$
\tau=2 \lambda \gamma p_{k}+N p_{k}
$$

and

$$
\varphi=N \gamma+\gamma^{2} \lambda-\frac{p_{k} \Omega N T}{K \log \left(1+\frac{\Gamma}{\lambda+N(10 P+\gamma)^{-1}}\right)} .
$$

Note that equation (17) is obtained by solving (16) for $x$. For this approximation, we ignore the first and second terms in (16) as third and fourth terms are more dominant. The third logarithmic terms is approximated by $\varphi$. This results in an expression that is of the quadratic form in $x$, which can be solved to obtain (17). Next, we provide the sum spectral efficiency performance of the FD-based MU MIMO system with the proposed training.

\section{NumERICAL RESUlTS}

We numerically evaluate the sum spectral efficiency (sum rate) of the FD-based MU MIMO system discussed in Section II. We compare the performance of the proposed sub-optimal CSI training length (16) with exhaustive search in (9) and fixed length CSI training. We set the value of $N_{0}=1$ and $\beta_{\mathrm{dt}}=$ 4. The value of residual SI is set to $\sigma_{\mathrm{SI}}^{2}=-10 \mathrm{~dB}$, unless specified otherwise. We use the function fzero provided by Matlab to solve (16) using the Brent's algorithm with $x_{i}=$ 0 and $x_{f}=50$. The sum spectral efficiency results in this section are produced by using equation (46) of [2].

In Fig. 2, we plot the sum spectral efficiency of the network with CSI training lengths based on the exhaustive search and proposed sub-optimal solution (16). The SNR is given by $P / N_{0}$. Here, we plot three cases: $M=16,32$ and 64 antennas against different values of SNR, with $K=8$ and $P=p_{k}=0$ $\mathrm{dB}$. The performance of the sub-optimal solution (16) matches closely with the exhaustive search method. However, it is seen that the performance of the sub-optimal scheme (16) is slightly lower than the exhaustive search scheme at low SNR, as the sub-optimal scheme allocates smaller training length, which in turn effects the CSI quality. From the results not presented here, we note that the requirement of CSI training gradually decreases with the increase in the number of antennas.

The sum spectral efficiency performance against various $K$ values is plotted in Fig. 3 with $T=600$ and $M=144$. We plot four different cases with $P=p_{k}=0 \mathrm{~dB}, 5 \mathrm{~dB}, 10 \mathrm{~dB}$ and $15 \mathrm{~dB}$. The spectral efficiency with the sub-optimal CSI training (16) is compared with the fixed length CSI training with $\beta_{\mathrm{ul}}=12$. We note that as $K$ increases, the proposed suboptimal scheme outperforms the fixed length CSI training and the performance gap becomes more dominant at high $P$ values. Moreover, it can be seen that when $P=0 \mathrm{~dB}$, the performance of the fixed training dominates. We also observed that at higher $K$ values, the CSI training length requirement decreases. This trend is due to the fact that the HD transmission interval, $T_{d}$, decreases as $K$ increases, therefore to compensate for large $K$ values, the proposed scheme reduces the CSI training length.

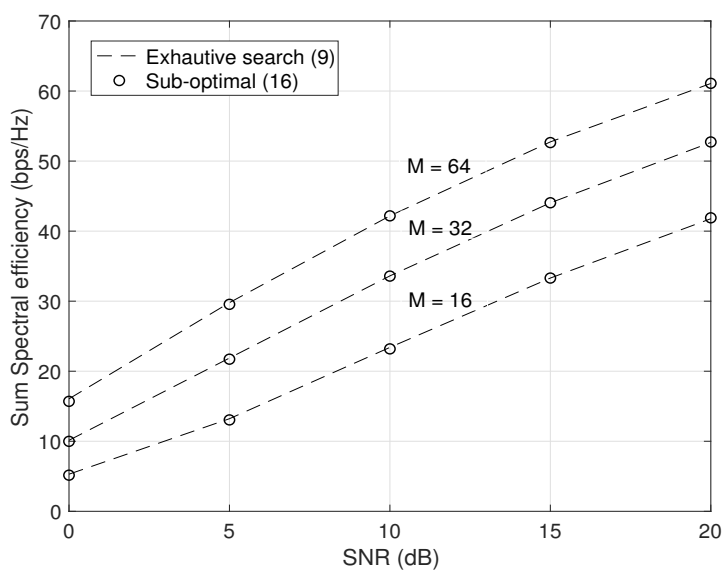

Fig. 2: Sum spectral efficiency vs. SNR.

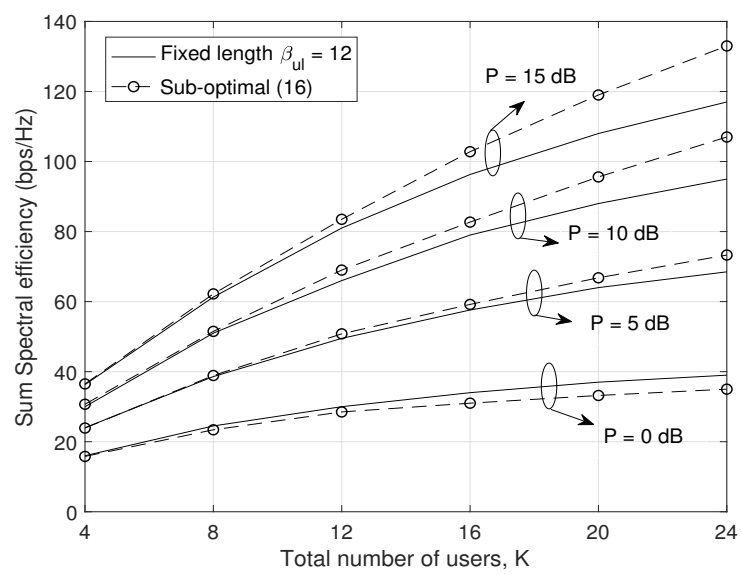

Fig. 3: Sum spectral efficiency vs. number of users $K$.

This is not possible with the fixed CSI training length scheme (16).

In Table I, we compute the normalized mean squared error (MSE) of the estimated channel and the associated sum spectral efficiency using the proposed sub-optimal scheme, open loop training scheme by $\mathrm{Du}$ et al. [1] and fixed length training [2]. The open loop UL training length [1] is given by

$$
\beta_{\mathrm{ul}}^{\mathrm{o}}=\sqrt{\frac{2(M-1) T}{\left(\Delta R^{\mathrm{INI}}+R^{\mathrm{ZF}}\right) M^{2}}},
$$

where $R^{\mathrm{ZF}}=\mathbb{E}\left[\log _{2}\left(1+P / M\left|\mathbf{h}_{k}^{H} \mathbf{v}_{k}\right|^{2}\right)\right]$. For large $T$, the value of $\Delta R^{\mathrm{INI}}$ becomes equal to $\log ((1+P) /(1+\bar{P}))$, where $\bar{P}=P /(1+P / M)[1]$. The normalized MSE of the estimated channel is given by

$$
\operatorname{MSE}(\hat{\mathbf{H}})=\frac{\|\mathbf{H}-\hat{\mathbf{H}}\|_{F}^{2}}{\|\mathbf{H}\|_{F}^{2}},
$$

where $\mathbf{H}=\left[\mathbf{h}_{1}, \ldots, \mathbf{h}_{K}\right]^{T}$. As the open-loop training scheme by $\mathrm{Du}$ et al. [1] is only applicable for $M=K$ configurations, therefore, for a fair comparison, we set $M=K=16$ and $T=1200$. It is seen that as the SNR increases, the MSE and sum spectral efficiency performance of the proposed sub- 
TABLE I: MSE of the estimated channel vs. SNR.

\begin{tabular}{|c|c|c|c|c|c|c|c|c|c|c|c|c|}
\hline \multirow[b]{3}{*}{ Schemes } & \multicolumn{12}{|c|}{ SNR (dB) } \\
\hline & \multicolumn{2}{|r|}{0} & \multicolumn{2}{|r|}{5} & \multicolumn{2}{|c|}{10} & \multicolumn{2}{|r|}{15} & \multicolumn{2}{|c|}{20} & \multicolumn{2}{|c|}{25} \\
\hline & $\begin{array}{l}\text { MSE } \\
(\mathrm{dB})\end{array}$ & $\begin{array}{c}R \\
\text { (bps/Hz) }\end{array}$ & $\begin{array}{l}\text { MSE } \\
(\mathrm{dB})\end{array}$ & $\begin{array}{c}R \\
\text { (bps/Hz) }\end{array}$ & $\begin{array}{l}\text { MSE } \\
(\mathrm{dB})\end{array}$ & $\begin{array}{c}R \\
\text { (bps/Hz) }\end{array}$ & $\begin{array}{l}\text { MSE } \\
(\mathrm{dB})\end{array}$ & $\begin{array}{c}R \\
\text { (bps/Hz) }\end{array}$ & $\begin{array}{l}\text { MSE } \\
(\mathrm{dB})\end{array}$ & $\begin{array}{c}R \\
\text { (bps/Hz) }\end{array}$ & $\begin{array}{l}\text { MSE } \\
(\mathrm{dB})\end{array}$ & $\begin{array}{c}R \\
\text { (bps/Hz) }\end{array}$ \\
\hline Du et al. [1] & -35.8 & 3.2 & -39.6 & 5.2 & -42.3 & 9.5 & -42.9 & 17 & -42.4 & 26.5 & -39.7 & 33.8 \\
\hline Sub-optimal (16) & -34.5 & 3 & -39.6 & 5.2 & -44.3 & 10.2 & -47.3 & 18.5 & -50.5 & 30.1 & -52.4 & 40.5 \\
\hline Fixed length & -24.8 & 1.6 & -34.1 & 4.3 & -41.4 & 9.6 & -45.8 & 18.2 & -47.7 & 28.5 & -48 & 39.4 \\
\hline
\end{tabular}

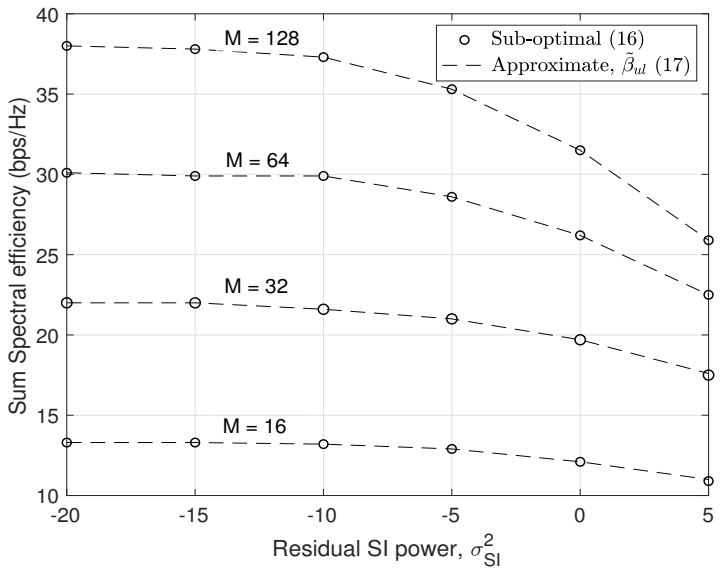

Fig. 4: Sum spectral efficiency vs. residual SI $\sigma_{\text {SI }}^{2}$.

optimal scheme becomes superior than the training scheme in Du et al. [1]. At low SNR values, the sum spectral efficiency performance with the fixed length training having $\beta_{\mathrm{ul}}=12$ is lower than the sub-optimal (16), as a large amount of training is required at low SNRs. However, we observe that as the SNR increases, the sum spectral efficiency performance of the fixed length training scheme improves and it outperforms Du et al. [1], as the latter fails to scale up the training requirement at high SNRs. We observe that the MSE performance increases with the increase in SNR values because this improves the channel estimation (3) at the $\mathrm{BS}$ as we have set $p_{k}=P$. However, for the scheme in Du et al. [1], the MSE performance improves till the $\mathrm{SNR}=15 \mathrm{~dB}$, then it gradually starts to decrease. Once again, the main reason for this trend is due to the fact that the optimal training duration in [1] decreases as the SNR increases. This decrease of $\beta_{\mathrm{ul}}^{\mathrm{o}}$ is also evident from (20), where, when $P \rightarrow \infty$, the terms $\Delta R^{\mathrm{INI}} \rightarrow \infty$ and $R^{\mathrm{ZF}} \rightarrow \infty$, hence resulting in $\beta_{\mathrm{ul}}^{\mathrm{o}} \rightarrow 0$. The main reason that the proposed sub-optimal scheme outperforms the other two schemes is due to its capability of scaling up the training requirement at both low and high SNR regimes. For example, the UL CSI training length with the proposed sub-optimal scheme are $33,21,16,14,16$ and 18 , for the SNR values of $0 \mathrm{~dB}, 5 \mathrm{~dB}, 10 \mathrm{~dB}, 15 \mathrm{~dB}, 20 \mathrm{~dB}$ and 25 respectively.

In Fig. 4, we plot sum spectral efficiency results for various residual SI power values, with $P=5 \mathrm{~dB}, T=500$ and $K=8$. Here, we compare the performance of the suboptimal scheme (16) and its approximate version (17). For all the antenna configurations, we observe a gradual decrease in the performance as the residual SI power increases. We also observe that the CSI training length requirement increases at higher $\sigma_{\text {SI }}^{2}$ values.

\section{CONCLUSION}

In this study, we have proposed sub-optimal solutions for the UL CSI training length that maximizes the spectral efficiency of MU MIMO systems with the FD-enabled BS. The sum spectral efficiency results obtained numerically show the effectiveness of the proposed UL training length compared to the fixed length CSI training. Contrary to the optimal training in HD systems [7], it is observed that the combined FD-HD transmission model studied in this paper is favourable for large antenna array configurations as it reduces the training overhead and increases the sum spectral efficiency of the system. For the future work, it will be useful to jointly optimize UL training and transmission power along with UL beamforming when users are equipped with multiple antennas.

\section{REFERENCES}

[1] X. Du, J. Tadrous, and A. Sabharwal, "Sequential beamforming for multiuser MIMO with full-duplex training," IEEE Trans. Wireless Commun., vol. 15 , no. 12, pp. 8551-8564, Dec 2016.

[2] J. Mirza, G. Zheng, K. Wong, S. Lambotharan, and L. Hanzo, "On the performance of multiuser MIMO systems relying on full-duplex CSI acquisition," IEEE Trans. Commun., vol. 66, no. 10, pp. 4563-4577, Oct 2018.

[3] G. C. Alexandropoulos and M. Duarte, "Low complexity full duplex MIMO: novel analog cancellation architectures and transceiver design." [Online]. Available: https://arxiv.org/pdf/1809.09474.pdf

[4] E. Everett, C. Shepard, L. Zhong, and A. Sabharwal, "SoftNull: manyantenna full-duplex wireless via digital beamforming," IEEE Trans. Wireless Commun., vol. 15, no. 12, pp. 8077-8092, Dec 2016.

[5] H. Iimori, G. Abreu, and G. C. Alexandropoulos, "Full-duplex transmission optimization for bi-directional MIMO links with QoS guarantees," in IEEE Global Conf. Sig. and Inf. Process., Anaheim, CA, USA, Nov 2018, pp. 201-205.

[6] M. Heino, D. Korpi, T. Huusari, E. Antonio-Rodriguez, S. Venkatasubramanian, T. Riihonen, L. Anttila, C. Icheln, K. Haneda, R. Wichman, and M. Valkama, "Recent advances in antenna design and interference cancellation algorithms for in-band full duplex relays," IEEE Commun. Mag., vol. 53, no. 5, pp. 91-101, May 2015.

[7] B. Hassibi and B. M. Hochwald, "How much training is needed in multiple-antenna wireless links?" IEEE Trans. Inf. Theory, vol. 49, no. 4 , pp. 951-963, April 2003.

[8] W. Santipach and M. L. Honig, "Capacity of beamforming with limited training and feedback," in IEEE Intl. Symp. on Inf. Theory, Seattle, WA, USA, July 2006, pp. 376-380.

[9] M. Kobayashi, N. Jindal, and G. Caire, "Training and feedback optimization for multiuser MIMO downlink," IEEE Trans. Commun., vol. 59, no. 8, pp. 2228-2240, Aug 2011.

[10] A. F. Dana, M. Sharif, and B. Hassibi, "On the capacity region of multiantenna gaussian broadcast channels with estimation error," in IEEE Intl. Symp. Inf. Theory, Seattle, WA, USA, July 2006, pp. 1851-1855. 
[11] G. Caire, N. Jindal, M. Kobayashi, and N. Ravindran, "Multiuser MIMO achievable rates with downlink training and channel state feedback," IEEE Trans. Inf. Theory, vol. 56, no. 6, pp. 2845-2866, June 2010. 